\title{
Sonidos del comer: propuestas para el estudio intermodal entre el sabor y la música
}

\author{
Eugenia Razumiejczyk ${ }^{1,2}$, Guillermo Macbeth ${ }^{1,2}$, Gisela Hurtado², \\ Carolina Pereyra Girardi ${ }^{3}$, Fernando Marmolejo-Ramos ${ }^{4, *}$ \\ ${ }^{1}$ Consejo Nacional de Investigaciones Científicas y Técnicas (CONICET) Argentina \\ ${ }^{2}$ Centro de Investigación y Transferencia de Entre Ríos (CITER) Argentina \\ ${ }^{3}$ Instituto de Investigaciones Cardiológicas (ININCA) Argentina \\ ${ }^{4}$ School of Psychology, The University of Adelaide, Australia
}

\begin{abstract}
Resumen
El objetivo de este artículo es proponer una síntesis original del proceso de correspondencias del gusto con otras modalidades sensoriales. Para ello, los autores presentan el concepto de integración multisensorial entendido como proceso de correspondencias y de suposición de la unidad, ambos facilitados por la congruencia semántica. Asimismo, se muestran diversos estudios que han investigado la percepción del gusto/sabor y sus correspondencias. Los principales resultados hallaron que parámetros específicos de sonidos pueden modificar la experiencia del gusto añadiendo significativamente placer a la experiencia de comer y beber del consumidor. Sin embargo, es importante destacar la relevancia de la validez ecológica en los estudios intermodales. Los autores presentan varias líneas de fructíferas de investigación. (C) 2018. Acad. Colomb. Cienc. Ex. Fis. Nat.
\end{abstract}

Palabras clave: Gusto; Sonido; Correspondencias; Integración; Intermodalidad; Percepción.

Sounds of eating: proposals for the crossmodal study between flavour and music

\begin{abstract}
The aim of this article is to propose an original synthesis of the correspondences between taste and other sensory modalities. For this, the authors present the concept of multisensory integration, understood as a process of correspondences and unity assumption, both facilitated by semantic congruence. Several studies that have investigated the taste/flavour perception and its correspondences are described. The main results found that specific sound parameters can modify the taste experience by adding significant pleasure to the consumer's eating and drinking experience. However, it is important to highlight the relevance of ecological validity in intermodal studies. The authors present several lines for future research. C 2018. Acad. Colomb. Cienc. Ex. Fis. Nat.
\end{abstract}

Key words: Taste; Sound; Correspondences; Integration; Crossmodality; Perception.

\section{Introducción}

Todas las modalidades sensoriales en el ser humano se encuentran integradas en el acto de comer y beber de forma tal que hacen que esa actividad aumente su intensidad hedónica. El individuo no sólo come para alimentarse o en situaciones sociales, sino también porque esta actividad le produce placer. Existen, en la actualidad, diversas ciencias y tecnologías que se han desarrollado para brindar más satisfacción en el acto de comer y beber.

Los sonidos se asocian al acto de comer de diversas maneras. Por un lado, se relacionan a la preparación de la comida, por ejemplo, al crujido de la sartén mientras fríe unas papas. Esto genera expectativas sobre el sabor que va a tener esta comida. Por otro lado, nuestra experiencia física mientras mordemos, masticamos o tragamos genera potencialmente información contextual sobre las claves del alimento e incluso información adicional somatosensorial proveniente de la sensación bucal. Por ejemplo, nos brinda información sobre cuán carbonatada es una bebida, cuan crocante es una galleta, cuan cremoso es un chocolate (Spence, 2016). De esta manera, un ambiente ruidoso o una música agradable de fondo mientras se está comiendo pueden influenciar positiva o negativamente al acto de comer (Spence, 2015); es decir, los sonidos pueden influir en la percepción del sabor.

En este sentido, Areni y Kim (1993) y Spence (2016) mostraron que los consumidores que escucharon música clásica en una tienda de vinos gastaron más que cuando escuchaban otro tipo de música. También se ha exhibido

\footnotetext{
*Correspondencia:

Fernando Marmolejo-Ramos, fernando.marmolejoramos@adelaide.edu.au Recibido: 1 de marzo de 2018

Aceptado: 7 de junio de 2018

Editor: Rubén Ardila
} 
en estudios de mercado (North, et al., 1999), que aquellos individuos que escuchaban música francesa en comparación con música alemana compraban más vinos franceses que alemanes, y viceversa. En términos de música clásica y su relación con el comportamiento de las personas frente a la comida, North, et al., (2003) mostraron que los sujetos gastaban $10 \%$ más mientras escuchaban música clásica ya que un ritmo rápido disminuye el tiempo de consumo (Milliman, 1986).

Estudios recientes han mostrado que parámetros específicos de sonidos pueden modificar la experiencia del gusto al agregar en forma significativa más placer a la experiencia de comer y beber del consumidor. Cuanto más gusta un sonido, más placer va a sentir la persona frente a un olor posteriormente presentado (Seo y Hummel, 2011). De esta manera, se produce una transferencia de un mecanismo activo, es decir, los sentimientos positivos que están asociados con la música son transferidos al placer asociado con la comida y bebida (Reinoso Carvalho, et al., 2016a; Reinoso Carvalho, et al., 2016b). No obstante, estos estudios se enfocaron en la selección de comidas y bebidas y no en su evaluación sensorial.

El objetivo de este artículo es proponer una síntesis original del proceso de correspondencia del sabor y otras modalidades sensoriales. Tales correspondencias pueden ser entendidas como transferencias de atributos desde una modalidad hacia otra modalidad. Por ejemplo, una música dulce refiere al gusto de un sonido, es decir que el atributo dulce se traslada desde la modalidad gustativa hacia la auditiva.

\section{La integración multisensorial}

La integración multisensorial ocurre cuando estímulos en diferentes modalidades se presentan al mismo tiempo (Spence, 2015). La coincidencia espacial también facilita la integración multisensorial bajo ciertas condiciones (Spence, 2013). Así, los observadores tendrán la tendencia a inferir que los elementos nuevos tienen un origen espaciotemporal común y construirán una única representación de un objeto o evento multisensorial (Bedford, 2001). Por ejemplo, en la situación del cine (Chen y Spence, 2017) escuchamos voces que parecen salir de los labios que se mueven de un actor y de quien inferimos habla a través de una pantalla.

Cuando dos estímulos sensoriales de diferentes modalidades ingresan al sistema cognitivo, nuestro sistema perceptual debe computar si éstos provienen de una causa común o si son emergentes de causas diferentes. De esta manera, los estímulos serán integrados o procesados por separado, respectivamente. La suposición de la unidad es la creencia del observador que dos o más claves unisensoriales provienen del mismo objeto (Chen y Vroomen, 2013). Tal creencia, por parte del observador, sirve como un modulador cognitivo de la integración multisensorial y es, a su vez, el mecanismo por el cual el cerebro humano resuelve los problemas intermodales. Así, las señales de diferentes sentidos son codificadas como una única representación de un objeto o evento (Spence, 2011).
La integración multisensorial se ve facilitada por la congruencia semántica. Ésta se refiere a aquellas situaciones en las que pares de estímulos auditivos y visuales son presentados y varían, esto es, coinciden o no coinciden en términos de su identidad y significado. Por ejemplo, en un experimento se presentan una palabra escrita y un sonido de una palabra que no coincide con la palabra escrita (van Atteveldt, et al., 2004). En general, se presume que estímulos visuales y auditivos son integrados cuando son semánticamente congruentes, así, se observarán algunas formas de mejora en el comportamiento de los participantes o en la actividad cerebral, esto es, menor tiempo de reacción en las respuestas o mayor taza de aciertos en identificación (Chen y Spence, 2010). Estudios recientes han investigado la congruencia semántica en otras modalidades sensoriales (Velasco, et al., 2016). Se ha estudiado la congruencia semántica entre las percepciones del sabor y palabras presentadas visualmente (Razumiejczyk, et al., 2013; Razumiejczyk, et al., 2015). Los resultados mostraron mayor facilitación intermodal en condición de congruencia, tanto en palabra completa, como incompleta y en anagramas.

La mayoría de los estudios en sus intentos de evaluar el impacto de la congruencia semántica en la percepción multisensorial han utilizado estímulos que, referidos a la misma categoría o concepto, no eran objetos o eventos que se podían hallar en la vida cotidiana de los participantes, es decir, los experimentos no poseían validez ecológica (Chen y Spence, 2017). Con la intención de incrementar la validez ecológica en tales experimentos, Razumiejczyk, et al., (2017) evaluaron la integración intermodal entre estímulos auditivos y del sabor en un experimento en el que se incorporó la participación de un experimentador que pronunciaba los estímulos auditivos en vez de un aparato auditivo. El experimentador se mantuvo constante entre los participantes y fue entrenado en el procedimiento para mantener constante también su desempeño en el procedimiento. Los resultados mostraron un menor tiempo de reacción y una mayor identificación en estímulos congruentes que en condiciones incongruentes y controles, en consistencia con estudios previos (Razumiejczyk, et al., 2015).

\section{Correspondencias intermodales}

Las correspondencias intermodales reflejan la tendencia a asociar rasgos o dimensiones de estímulos aparentemente arbitrarios a través de los sentidos (Spence y Deroy, 2013). En tal caso, se asocian atributos o características que permanecen juntos, aunque no se tenga la creencia de que ellos coocurren juntos en el mismo objeto (Chen y Spence, 2017), a diferencia de la suposición de la unidad. Lo distintivo de las correspondencias intermodales es que operan independientemente de la suposición de la unidad y están sujetas a objetos que siguen siendo percibidos como distintos (Deroy y Spence, 2016). Las correspondencias son un tipo de interacción entre los sentidos, esto es, no requieren que los sentidos involucren el objeto o propiedad y puede ocurrir 
incluso cuando dos objetos son percibidos como separados en el espacio y en el tiempo (Deroy y Spence, 2016). Esta capacidad puede explicar por qué las correspondencias se vuelven tan importantes en la adquisición del lenguaje en el sentido de que colaboran con el recuerdo y el establecimiento de la relación entre objetos, es decir, palabra oral y referencia visual (Matusz, et al., 2017).

El origen de las correspondencias es diverso. Se pueden distinguir que algunas correspondencias surgen de las regularidades del ambiente, por ejemplo, la correspondencia entre el sonido y el tamaño, o el sonido y la elevación. Otras se originan en percepciones estructurales similares, como por ejemplo, brillo y volumen. Otras correspondencias se originan en similitudes semánticas o hedónicas (Deroy y Spence, 2016).

Estos fenómenos se han venido estudiando desde hace más de 80 años. Sapir (1929) observó una correspondencia entre los sonidos vocales " $i$ " y "a" y el tamaño de los objetos. La palabra sin sentido en inglés mal era asociada a objetos grandes, mientras que mil era asociada a objetos pequeños. Asimismo, la palabra maluma, era asociada a formas redondeadas y la palabra takete, a formas puntiagudas (Köhler, 1929). Se han estudiado las correspondencias entre las dimensiones sensoriales tales como timbres musicales y olores, formas y colores entre otros (Spence y Deroym, 2013). Se ha hallado que las correspondencias permanecen constantes entre los dominios sensoriales, esto es, la correspondencia entre formas y nombres permanece constante con formas y nombres de olores, gustos y sabores (Spence y Ngo, 2012). En estos casos, olores frutales y sabores dulces coincidieron con la palabra maluma y con formas redondeadas.

En la Figura 1 se esquematiza el procesamiento de los estímulos en la integración intermodal.

\section{Influencias de las correspondencias en la percepción del gusto/sabor}

Diversos estudios han investigado la percepción del gusto/ sabor y sus correspondencias. Tales estudios han hallado que el gusto dulce se corresponde con formas redondeadas y que los gustos salados, amargos y ácidos, con formas puntiagudas (Velasco, et al., 2016). En este sentido, se ha estudiado que el envoltorio de los productos alimenticios puede influir en las expectativas de los gustos del contenido; esto es, las personas buscan paquetes redondeados para gustos dulces, así como diseños angulares para gustos más ácidos (Lunardo y Livat, 2016). Asimismo, el sonido puede añadir un valor significativo, y placentero, a la experiencia del consumidor (Reinoso Carvalho, et al., 2016).

Estudios recientes han identificado asociaciones entre percepciones del sabor y auditivas. Crisinel y Spence (2009) hallaron una asociación entre el tono de los sonidos y los gustos amargos y ácidos. Los resultados mostraron una correspondencia entre tonos graves y gustos amargos y entre tonos agudos y gustos ácidos, aunque en sus experimentos sólo nombraban los gustos y no los degustaban. Los autores explicaron el origen de estas asociaciones como señales de peligro. El gusto amargo se encuentra, en general, en los venenos y el gusto ácido refleja bajos niveles de $\mathrm{pH}$ en las sustancias. Los tonos agudos se asocian a alarmas y los tonos graves a objetos grandes (Parise y Spence, 2009). Los tonos graves pueden ser señal de peligro, por ejemplo, cuando se acerca un predador.

Mesz, et al., (2011) investigaron si los gustos básicos dulce, salado, amargo y ácido son asociados con parámetros en la producción musical inducidos por estos. Para ello, solicitaron a músicos expertos que improvisaran piezas musicales basadas en estas palabras y en un segundo experimento estudiaron si esas improvisaciones eran consistentes con las palabras de los gustos estudiados en la población general sin entrenamiento específico en música. Los resultados generales mostraron que las improvisaciones duraron 43,3 segundos en promedio. Asimismo, el gusto más placentero, dulce (Moskowitz, et al., 1974), se relacionó con altos valores de placeres psicoacústicos, que corresponden con la intensidad sonora suave y de baja rugosidad. La palabra ácido se relacionó con improvisaciones agudas, fuertes y disonantes que se corresponden con altos valores de agudeza sensorial.

Posteriormente, Crisinel Cosser, et al., (2012) estudiaron la influencia de la música en la evaluación de cuan dulce o amargo era un caramelo agridulce. Con la ayuda de una banda sonora produjeron piezas musicales dulces y

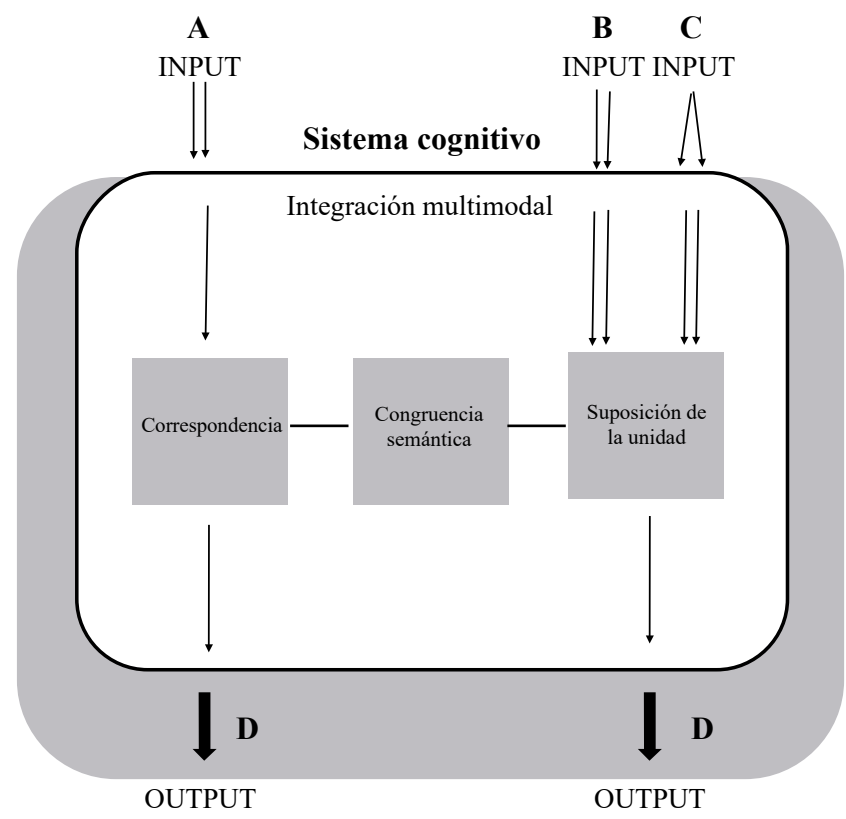

Figura 1. Integración intermodal: correspondencia, congruencia semántica y suposición de la unidad. A. Los estímulos provienen de un mismo tiempo, pero de objetos diferentes. B. Los estímulos provienen de un tiempo común, aunque de un objeto diferente. $\mathbf{C}$. Los estímulos provienen simultáneamente de un mismo objeto. D. Las señales provenientes de diferentes sentidos son codificadas como una única representación de un objeto o de un evento. 
amargas. Las piezas musicales amargas contenían predominantemente tonos bajos mientras que las dulces estaban compuestas por tonos altos (Crisinel y Spence, 2009; Mesz, et al., 2011). Los resultados mostraron que los participantes que evaluaron el caramelo mientras escucharon la pieza de música dulce respondieron entre $5 \%$ y $10 \%$ más dulce que aquellos que escucharon música amarga. Estos hallazgos muestran que es posible utilizar la música para crear un percepto gustativo que no se encuentra presente en el acto de comer.

Los estudios previos se enfocan exclusivamente en el gusto. Sin embargo, en la construcción de la percepción del sabor se produce una integración intermodal entre la sensación del gusto y la sensación del olfato (Spence, 2013). Por esta razón, Reinoso Carvalho, et al., (2016) quisieron estudiar si el sonido podía influenciar los atributos del sabor también, tales como la cremosidad y aspereza del chocolate. Los resultados mostraron que las piezas musicales cremosas elevaron los puntajes de cremosidad del chocolate en comparación con las piezas musicales ásperas que potencialmente disminuían la cremosidad percibida en éstos. Los resultados concluyeron que los sonidos tienen un efecto perceptual en la comida sin alterar su experiencia hedónica independientemente del hecho de que algunas personas prefieran algunos sonidos sobre otros (Wang y Spence, 2015). Del mismo modo, Reinoso Carvalho, et al., (2016) estudiaron la influencia de la música y de la etiqueta de una cerveza con relación al gusto de una cerveza. Un primer grupo bebió la cerveza sin la etiqueta, el segundo grupo lo hizo con la etiqueta y el tercer grupo, con la etiqueta y con música (un fragmento de la canción Oceans of light). Los resultados mostraron que a las personas les tiende a agradar más la cerveza cuando la experiencia de interacción entre la música y el gusto es positivo y claro. En este sentido, los resultados fueron coherentes con los de Kantono, et al., (2016) quienes reportaron que escuchar música puede influenciar a la percepción hedónica de la comida y la bebida.

\section{Conclusiones}

El ser humano no sólo se alimenta para vivir, sino que además ha creado tecnologías para aumentar su intensidad hedónica (por ejemplo, intensificando el color de algunos alimentos y bebidas). También ha agregado más intensidad a otros sentidos complementarios, como es el caso de bebidas carbonatadas o alimentos crocantes cuyo crujido indica su cualidad, o incluso la música que acompaña los restaurantes y supermercados (Crisinel, et al., 2012). Así, resulta posible utilizar la música para aumentar o disminuir la intensidad de un sabor. Del mismo modo, a nivel visual se ha estudiado que el envoltorio de los productos alimenticios puede influir en las expectativas de los gustos del contenido. Es decir, las personas buscan paquetes redondeados para gustos dulces y diseños angulares para gustos más ácidos. De este modo, puede afirmarse que el sabor es una integración de información que proviene de diversos canales sensoriales. Es posible utilizar recursos tecnológicos para operar sobre tal integración.
Es importante destacar la relevancia de la validez ecológica en los estudios intermodales. En tal sentido, hemos realizado aportes previos que pueden ser considerados complementarios con esta revisión (Razumiejczyk, et al., 2017).

Del análisis del estado actual de este campo específico de estudios se pueden derivar varias líneas fructíferas de investigación. Algunas preguntas que podrían guiar futuros estudios son: ¿cuáles son los estímulos auditivos ecológicamente adecuados para formar parte de un experimento intermodal entre la audición y el sabor?, ¿es posible que una música dulce haga más dulce un sabor?, ¿es posible intensificar también el gusto amargo mediante la misma estrategia experimental?, ¿se puede reducir la intensidad de un gusto dulce mediante la exposición simultánea a una música amarga? Por su potencial aplicación es también relevante un conjunto de preguntas que relaciona los efectos intermodales de la música sobre la visión, por ejemplo, ¿puede una música dulce intensificar el sabor dulce a partir de la observación de la foto de un alimento dulce?, ¿se puede replicar este fenómeno intermodal con el gusto amargo a partir de música y fotos?

\section{Conflicto de intereses}

Los autores declaran que no existe conflicto de intereses en torno al desarrollo de la investigación.

\section{Contribución de los autores}

Los autores declaran que contribuyeron en igual medida en este trabajo.

\section{Referencias}

Areni, C. S., Kim, D. (1993). The influence of background music on shopping behaviour: classical versus top-forty music in a wine store. Advances in Consumer Research. 20: 336-340.

Bedford, F. (2001). Towards a general law of numerical/object identity. Current Psychology of Cognition. 20: 113-176.

Chen, L., Vroomen, J. (2013). Intersensory binding across space and time: a tutorial review. Attention, Perception and Psychophysics. 75 (5): 790. Disponible en http://dx.doi. org/10.3758/s13414-013-0475-4

Chen, Y. -C., Spence, C. (2010). When hearing the bark helps to identify the dog: Semantically-congruent sounds modulate the identification of masked pictures. Cognition. 114 (3): 389-404. Disponible en: http://dx.doi.org/10.1016/j. cognition.2009.10.012

Chen, Y. -C., Spence, C. (2017). Assessing the role of the 'unity assumption' on multisensory integration: A review. Frontiers in Psychology, 8. Disponible en: http://dx.doi.org/10.3389/ fpsyg.2017.00445

Crisinel, A. S., Spence, C. (2009). Implicit association between basic tastes and pitch. Neuroscience letters. 464 (1): 3942. Disponible en: http://dx.doi.org/10.1016/j.neulet.2009. 08.016

Crisinel, A. S., Cosser, S., King, S., Jones, R., Petrie, J., Spence, C. (2012). A bittersweet symphony: Systematically modulating the taste of food by changing the sonic properties of the soundtrack playing in the background. Food Quality and Preference. 24 (1): 201-204. Disponible en: http://dx.doi. org/10.1016/j.foodqual.2011.08.009 
Deroy, O., Spence, C. (2016). Crossmodal correspondences: four challenges. Multisensory research. 29 (1-3): 29-48. Disponible en: http://dx.doi.org/10.1163/22134808-00002488

Kantono, K., Hamid, N., Shepherd, D., Yoo, M. J., Grazioli, G., Carr, B. T. (2016). Listening to music can influence hedonic and sensory perceptions of gelati. Appetite. 100: 244-255. Disponible en: http://dx.doi.org/10.1016/j.appet. 2016.02.143

Köhler, W. (1929). Gestalt Psychology. New York, Estados Unidos: Liveright.

Lunardo, R., Livat, F. (2016). Congruency between colour and shape of the front labels of wine: effects on fluency and aroma and quality perceptions. International Journal of Entrepreneurship and Small Business. 29 (4): 528-541. Disponible en: http://dx.doi.org/10.1504/IJESB.2016.10000516

Matusz, P. J., Wallace, M. T., Murray, M. M. (2017). A multisensory perspective on object memory. Neuropsychologia. 105: 243. Disponible en: http://dx.doi.org/10.1016/j. neuropsychologia.2017.04.008

Mesz, B., Trevisan, M. A., Sigman, M. (2011). The taste of music. Perception. 40 (2): 209-219. Disponible en: http:// dx.doi.org/10.1068/p6801

Milliman, R.E. (1986). The influence of background music on the behavior of restaurant patrons. Journal of Consumer Research. 13 (2): 286-289. Disponible en: http://dx.doi. org/10.1086/209068

Moskowitz, H. R., Kluter, R. A., Westerling, J., Jacobs, H. L. (1974). Sugar sweetness and pleasantness: evidence for different psychological laws. Science. 184 (4136): 583585. Disponible en: http://dx.doi.org/10.1126/science.184. 4136.583

North, A. C., Hargreaves D. J., McKerndrick, J. (1999). The influence of in-store music on wine selections. Journal of Applied Psychology. 84 (2): 271-276. Disponible en: http:// dx.doi.org/10.1037/0021-9010.84.2.271

North, A. C., Shilcock, A., Hargreaves, D. J. (2003). The effect of musicalstyleonrestaurantcustomers'spending.Environment and behavior. 35 (5): 712-718. Disponible en: http://dx.doi. org/10.1177/0013916503254749.

Parise, C.V., Spence, C. (2009). When birds of a feather flock together: synesthetic correspondences modulate audiovisual integration in non-synesthetes. PloS ONE. 4: e5664. Disponible en: http://dx.doi.org/10.1371/journal. pone. 0005664

Razumiejczyk, E., Macbeth, G., Leibovich de Figueroa, N. (2013). Interferencia entre el procesamiento de estímulos gustativos y estímulos visuales lingüísticos incompletos en la memoria operativa. Revista Latinoamericana de Psicología. 45: 231-239

Razumiejczyk, E., Macbeth, G., Marmolejo-Ramos, F., Noguchi, K. (2015). Crossmodal integration between visual linguistic information and flavour perception. Appetite. 91: 76-82. Disponible en: http://dx.doi.org/10.1016/j.appet.2015.03.035

Razumiejczyk, E., Pereyra Girardi, C., Crivello, M. C., Fioramonti, M., Macbeth, G., Marmolejo Ramos, F. (2017). Crossmodal interference between language and flavour. Revista Latinoamericana de Psicología. 49 (2): 91-101. Disponible en: http://dx.doi.org/10.1016/j.rlp.2016. 01.002
Reinoso Carvalho, F. R., Velasco, C., van Ee, R., Leboeuf, Y., Spence, C. (2016a). Music influences hedonic and taste ratings in beer. Frontiers in psychology, 7. Disponible en: http://dx.doi.org/10.3389/fpsyg.2016.00636

Reinoso Carvalho, F. R., Wang, Q. J., Van Ee, R., Spence, C. (2016) b. The influence of soundscapes on the perception and evaluation of beers. Food Quality and Preference. 52: 32-41. Disponible en: http://dx.doi.org/10.1016/j.foodqual. 2016.03.009

Sapir, E. (1929). A study in phonetic symbolism. Journal of Experimental Psychology. 12 (3): 225. Disponible en: http://dx.doi.org/10.1037/h0070931

Seo, H. S., Hummel, T. (2011). Auditory-olfactory integration: Congruent or pleasant sounds amplify odour pleasantness. Chemical Senses. 36 (3): 301-309. Disponible en: http:// dx.doi.org/10.1093/chemse/bjq129

Spence, C. (2011). Crossmodal correspondences: A tutorial review. Attention, Perception, \& Psychophysics. 73 (4): 971-995. Disponible en: http://dx.doi.org/10.3758/s13414010-0073-7

Spence, C. (2013). Just how important is spatial coincidence to multisensory integration? Evaluating the spatial rule. Annals of the New York Academy of Sciences. 1296 (1): 31-49. Disponible en: http://dx.doi.org/10.1111/nyas.12121

Spence, C. (2015). Multisensory flavor perception. Cell. 161 (1): 24-35. Disponible en: http://dx.doi.org/10.1016/j.cell.2015. 03.007

Spence, C. (2016). Sound - the forgotten flavour sense, En B. Piqueras-Fiszman y C. Spence (Eds.), Fundamental Neuroscience Through to the Marketplace (pp. 81-105). Cambridge, Reino Unido: Elsevier.

Spence, C., Deroy, O. (2013). How automatic are crossmodal correspondences?. Consciousness and cognition. 22 (1): 245-260. Disponible en: http://dx.doi.org/10.1016/j.concog. 2012.12.006

Spence, C., Ngo, M. K. (2012). Assessing the shape symbolism of the taste, flavour, and texture of foods and beverages. Flavour. 1 (1): 12. Disponible en: http://dx.doi. org/10.1186/2044-7248-1-12

van Atteveldt, N., Formisano, E., Goebel, R., Blomert, L. (2004). Integration of letters and speech sounds in the human brain. Neuron. 43 (2): 271-282. Disponible en: http://dx.doi.org/10.1016/j.neuron.2004.06.025

Velasco, C., Salgado-Montejo, A., Elliot, A. J., Woods, A. T., Alvarado, J., Spence, C. (2016). The shapes associated with approach/avoidance words. Motivation and Emotion. 40 (5): 689-702. Disponible en: http://dx.doi.org/10.1007/ s11031-016-9559-5

Velasco, C., Woods, A. T., Petit, O., Cheok, A. D., Spence, C. (2016). Crossmodal correspondences between taste and shape, and their implications for product packaging: a review. Food Quality and Preference. 52: 17-26. Disponible en: http://dx.doi.org/10.1016/j.foodqual.2016.03.005

Wang, Q., Spence, C. (2015). Assessing the effect of musical congruency on wine tasting in a live performance setting. i-Perception. 6 (3). Disponible en: http://dx.doi. org/10.1177/2041669515593027 